\title{
TERAPIA NUTRICIONAL NO TRANSPLANTE HEPÁTICO
}

\author{
Mônica Beatriz PAROLIN, Francisca Eugênia ZAINA e \\ Reginaldo Werneck LOPES
}

RESUMO - Racional - Deficiências nutricionais, por vezes graves, são comuns em pacientes com insuficiência hepática, candidatos a transplante de fígado. A terapia nutricional pode corrigir total ou parcialmente tais deficiências, melhorando as condições clínicas e o prognóstico desses indivíduos, frente ao grande desafio do transplante hepático. Objetivos - Breve revisão do papel do fígado no metabolismo dos diversos nutrientes. Descrição dos métodos de avaliação do estado nutricional, traçando-se as bases da terapia nutricional segundo condições hepáticas diversas, no pré e pós-transplante, em relação às necessidades calóricas e dos diversos nutrientes. Apresentação de intervenções nutricionais, no controle das complicações metabólicas resultantes do uso de drogas imunossupressoras. Conclusão - A terapia nutricional é valiosa aliada no tratamento clínico de pacientes candidatos ou já submetidos ao transplante hepático, contribuindo para um prognóstico favorável e para a melhora da qualidade de vida desses indivíduos.

DESCRITORES - Transplante de fígado. Insuficiências hepática, dietoterapia. Hepatopatias, dietoterapia. Dietoterapia. Estado nutricional.

\section{INTRODUÇÃO}

Adequada avaliação do estado nutricional em pacientes portadores de hepatopatias crônicas permite diagnosticar importantes desvios e enseja a aplicação de medidas de correção capazes de melhorar o prognóstico, especialmente quando se pretende submeter tais pacientes ao recurso heróico do transplante hepático.

A terapia nutricional representa um dos procedimentos de maior importância no manejo das doenças do fígado, devendo ser considerada como um adjuvante imprescindível às opções terapêuticas de que dispõe a clínica.

Moléculas de nutrientes provenientes da digestão, absorvidas em nível de enterócitos e conduzidas através da circulação portal, encontram no fígado um complexo laboratório de transformação, armazenagem e redistribuição dos metabólitos destinados à nutrição dos órgãos periféricos e à própria síntese macromolecular hepática. Provenientes de outros tecidos, demais nutrientes vêm ao fígado, como os ácidos graxos e o glicerol originários dos tecidos adiposos, e importantes contribuintes para o provimento energético.
Assim, também, são nutrientes de afluxo hepático o lactato e o piruvato originados nas células sangüíneas e nos músculos esqueléticos, a alanina, outros aminoácidos e os alfa-cetoácidos ramificados, resultantes da transaminação da leucina, isoleucina e valina.

Através da glicogenólise do glicogênio armazenado, o fígado garante a produção de glicose e, portanto, a energia necessária aos processos metabólicos dos demais tecidos. Outro importante substrato, o acetoacetato, se produz pela oxidação dos ácidos graxos provenientes da gordura alimentar. Lipídios de depósito, como os fosfolipídios e o triacilglicerol são também sintetizados pelo fígado para armazenagem como lipoproteínas e sua utilização é gradativa, segundo as exigências dos tecidos periféricos ${ }^{(34)}$. Podemos idealizar o fígado como uma central metabólica provida de sensores que identificam as necessidades energéticas que ocorrem nos tecidos.

Pela atividade hepática e renal se produz carnitina, nutriente necessário para a performance adequada dos músculos esqueléticos e do cardíaco, tecidos estes capazes de gerar energia própria pela utilização metabólica de ácidos graxos.

Serviço de Transplante Hepático, Hospital de Clínicas da Universidade Federal do Paraná, Curitiba, PR.

Endereço para correspondência: Dra. Mônica Beatriz Parolin - Rua Lamenha Lins, 2280 - 80220-080 - Curitiba, PR. e-mail: mbparolin@ hotmail.com 
A regulação da relação ATP/ADP é garantida pela presença de creatina, cuja síntese final também se processa no fígado.

Freqüentemente, proteínas sintetizadas pelo fígado estão reduzidas em pacientes portadores de hepatopatias. Clinicamente essas deficiências se evidenciam pelo decréscimo dos respectivos valores no sangue circulante, como a albumina, a protrombina, a ceruloplasmina, a transferrina e a proteína que se liga ao retinol. Entre os aminoácidos importantes para a integridade muscular a glutamina é considerada como essencial; sua síntese está aumentada nos processos catabólicos e ela representa $61 \%$ dos aminoácidos constituintes da massa muscular. Entre outros nutrientes importantes que devem ser considerados na correção da deficiência nutricional destacam-se a arginina, pelo seu poder de geração de óxido nítrico, a colina, cistina, taurina e tirosina que necessitam de reposição porque, nas hepatopatias, sua síntese está prejudicada e a insulina e o glucagon, elementos considerados de fundamental importância no crescimento do parênquima hepático e na integridade da mucosa intestinal, onde a produção de IgA representa uma das garantias da integridade imunológica ${ }^{(28)}$.

Da mesma forma, a doença hepática crônica cursa com deficiência das vitaminas hidrossolúveis, tiamina, piridoxina e ácido fólico, especialmente se o fator etiológico em jogo for o álcool.

Quanto às vitaminas lipossolúveis, a vitamina $\mathrm{A}$, que geralmente é transportada sob a forma de um complexo de ligação do retinol à pré-albumina, nas hepatopatias crônicas se ressente da síntese da proteína de ligação e da adequada formação de pré-albumina transportadora.

Já a vitamina $\mathrm{D}$ depende do metabolismo hepático para a produção de 25-hidroxi-vitamina $\mathrm{D}$, que no rim se transforma no composto ativo 1,25-hidroxi-vitamina $\mathrm{D}$. Nas hepatopatias alcoólicas diminui a formação renal de hidroxilase necessária para a síntese de vitamina D ativa ou se forma uma hidroxilase anômala (D-24), capaz de gerar um metabólito inativo de vitamina $\mathrm{D}$.

Nas hepatopatias que cursam com colestase ou nas condições em que a falta de secreção biliar impede a formação de quilomicrons de metaquinona e filoquinonas, quando a fonte é alimentar poder-se-á constatar deficiência de vitamina $\mathrm{K}$, especialmente por defeito no processo absortivo ${ }^{(29)}$.

Assim, foram relacionados apenas alguns dos fundamentais micronutrientes que nas hepatopatias crônicas têm seu metabolismo prejudicado, o que determina insidiosa e progressiva deterioração do estado nutricional dos pacientes. Fator agravante em mais de $30 \%$ dos casos de cirrose é o hipermetabolismo, que pode ser explicado pelo incremento de atividade $\beta$-adrenérgica. Essa condição, entretanto, parece ser de natureza extra-hepática, pois persiste por mais de 1 ano após o transplante ${ }^{(24)}$.

Os pacientes hipermetabólicos apresentam, em geral, maior gasto energético em repouso, o que contribui ainda mais para o estado de carência nutricional.
Na evolução da doença hepática apresentam-se, gradativamente, outras condições que propiciam a desnutrição, como a anorexia e a náusea que redundam em diminuição da ingestão de alimentos ou a síndrome disabsortiva que resulta ora da deficiência de sais biliares e enzimas pancreáticas, ora da esterilização da flora intestinal pelo emprego da neomicina, nos casos de encefalopatia iminente.

Maior dispêndio energético em repouso se observa como outro elemento agravante da desnutrição, especialmente nos casos em que o fator etiológico é o álcool e na situação não incomum de ocorrência de sepse. Figuram ainda como fatores predisponentes para a desnutrição nas hepatopatias crônicas as alterações verificadas no metabolismo dos carboidratos, proteínas e gorduras que redundam em resistência periférica à insulina, redução das reservas hepáticas de glicogênio, maior aproveitamento das gorduras para a produção de energia e um aumento do catabolismo protéico, com grave perda da massa celular corpórea, esta parecendo estar ligada a menor produção do fator de crescimento insulina-símile (IGF-1) ${ }^{(3)}$.

Em $70 \%$ a $100 \%$ dos casos de doença hepática terminal a desnutrição protéico-calórica (DPC) faz parte da grande síndrome que caracteriza o quadro dramático que se busca aliviar pelo transplante hepático ${ }^{(10)}$. Sua correta avaliação e correção revestem-se de extrema importância para a garantia de funcionamento do enxerto, representando pois, fator prognóstico que precisa ser obrigatoriamente considerado por suas implicações na morbidade e mortalidade. Quanto ao prognóstico relacionado ao estado nutricional, MÜLLER et al. ${ }^{(23)}$ constataram que os índices de mortalidade não são significativamente diferentes em portadores de hepatopatias crônicas com vários graus de desnutrição, mas em relação aos resultados do transplante hepático houve marcante melhoria de sobrevida ( $83 \%$ versus $47 \%$ ) entre os que apresentavam melhor estado nutricional na época do transplante. PIKUL et al. ${ }^{(31)}$, avaliando retrospectivamente o efeito da DPC na morbidade e mortalidade pós-operatória de 68 receptores de transplante hepático, diagnosticaram a presença de desnutrição em $79 \%$ dos pacientes. A desnutrição moderada ou grave associou-se à necessidade de ventilação mecânica por tempo mais prolongado, havendo maior incidência de traqueostomias e o tempo de permanência em UTI e hospitalar foi mais longo. Além disso, esses autores observaram tendência a maior mortalidade nos pacientes desnutridos. Em estudo semelhante, HASSE et al.$^{\left({ }^{(8)}\right.}$ detectaram a presença de DPC em $70 \%$ de 500 pacientes submetidos a transplante hepático e confirmaram que pacientes desnutridos apresentam períodos mais prolongados de permanência em UTI e de internação hospitalar, quando comparados aos que exibem bom estado nutricional.

A DPC afeta de modo adverso os resultados do transplante hepático porque prolonga a permanência hospitalar, eleva os custos do procedimento e aumenta a morbidade em virtude de seus efeitos deletérios sobre a função imunológica, propiciando infecção e retardando o processo de cicatrização. Felizmente, seu impacto negativo 
pode ser minimizado pela intervenção nutricional na fase de pré e pós-transplante. A terapia nutricional ofertada ao candidato a transplante hepático visa prover quantidades adequadas de calorias, proteínas, vitaminas, fluidos e eletrólitos, sem precipitar ou agravar condições como a encefalopatia hepática e a ascite. Os benefícios dessa abordagem na fase pré-transplante incluem melhora da defesa imunológica, da cicatrização das feridas e a recomposição das reservas nutricionais $^{(1,4,10,15,17,18,31,32) \text {. }}$

Embora menos freqüente que a DPC, a obesidade pode estar presente em portadores de doença hepática crônica avançada. Estudos recentes mostram que pacientes com obesidade grave têm risco maior de complicações perioperatórias tais como infecção de parede, diabetes mellitus, hipertensão arterial, infecção respiratória e insuficiência respiratória ${ }^{(16)}$

\section{PARÂMETROS DE AVALIAÇÃO DO ESTADO NUTRICIONAL}

A avaliação do estado nutricional (EN) tanto nos candidatos, como nos que já foram submetidos ao transplante hepático representa um desafio para a equipe multidisciplinar, pela presença de alterações que decorrem da própria hepatopatia crônica, aumentando a limitação dos métodos usuais utilizados. Por isto, exige-se atenção especial do nutricionista, que deve utilizar o maior número possível de parâmetros a fim de aumentar a confiabilidade dos resultados. Há que não esquecer a principal utilidade dessa análise que é a do controle evolutivo do EN a médio e longo prazo. A DPC é achado comum em pacientes com doença hepática avançada, candidatos a transplante e deve ser considerada quando as medidas antropométricas se situam abaixo dos padrões de normalidade para cada parâmetro. Sua origem é multifatorial, colaborando intercorrências comumente observáveis nas hepatopatias crônicas ${ }^{(18,20,21)}$. A presença de ascite, cujo efeito compressivo contribui para a saciedade precoce, a gastrite, hemorragias digestivas, encefalopatia e sepse representam fatores prejudiciais à adequada ingestão quantitativa de nutrientes. Outras vezes, a dieta é qualitativamente deficiente em vista de medidas terapêuticas dirigidas às complicações da hepatopatia, como é o caso da falta de administração de proteínas na encefalopatia, de sódio e de fluidos no edema e na ascite ou de gorduras na presença de má absorção, por deficiência pancreática, biliar ou por enteropatia. Perda de peso (60\%), náusea $(55 \%)$ e anorexia $(87 \%)^{(18,20,21)}$, intercorrências constantes nas hepatopatias, concorrem para a instalação da DPC. Faltam estudos sobre a DPC em pré-cirróticos, mas em cirróticos alcoólicos a prevalência é de $34 \%$ a $82 \%$, podendo chegar a $100 \%$ em pacientes hospitalizados. Em não-alcoólicos a prevalência oscila entre $27 \%$ e $87 \%{ }^{(4)}$. Efeitos da terapia medicamentosa comumente empregada, como a neomicina, a lactulose, a colestiramina e os diuréticos podem traduzirse por atrofia das vilosidades e diarréia, como também pelo prejuízo na absorção de folatos, zinco, potássio, magnésio e vitaminas lipossolúveis. Nos pacientes hospitalizados somam-se outros fatores que merecem atenta consideração, como jejuns prolongados e repetidos para procedimentos diagnósticos, retirada constante de sangue para análises bioquímicas, hemorragia digestiva e alterações neuropsiquiátricas. Vale considerar que cada procedimento de paracentese descompressiva para retirada de líquido ascítico pode espoliar de 50 a $100 \mathrm{~g}$ de proteínas.

\section{Necessidades nutricionais e energéticas}

O maior objetivo da avaliação do EN e correção das deficiências em candidatos ao transplante é a melhoria da qualidade de vida até a cirurgia, pelo estabelecimento de reservas nutricionais e metabólicas capazes de garantir a síntese protéica e a regeneração das células hepáticas após o trauma cirúrgico. Condições nutricionais adequadas garantem também o processo de cicatrização e a competência do sistema imunitário na prevenção de infecções. Deve-se evitar dietas muito restritivas para que a provisão de nutrientes seja adequada em quantidade e qualidade, respeitando-se naturalmente, as restrições que se precisa aplicar a pacientes com encefalopatia hepática, já que apenas pequena parcela $(5 \%$ a 7\%) apresenta intolerância às proteínas, necessitando de restrição.

A dieta deve também corrigir alterações eletrolíticas, deficiências de vitaminas e minerais, minimizando o acúmulo de sódio e fluidos. Visa, ainda, limitar o catabolismo, tentando manter um balanço nitrogenado positivo e, acima de tudo, deve evitar que seja fonte de complicações.

Depois do transplante os mesmos objetivos devem ser perseguidos, com vistas à preservação e recuperação do enxerto após a isquemia.

A maioria dos estudos sobre cirróticos relata que suas exigências energéticas estão aumentadas, especialmente quando portadores de ascite de volume moderado a grande. Após as paracenteses há diminuição do gasto energético, o que sugere possível relação da ascite com as necessidades energéticas ${ }^{(5,11)}$.

Na prática clínica, a determinação das necessidades energéticas pode ser feita através da equação de Harris-Benedict que apresenta coeficientes distintos de peso, altura e idade, segundo os sexos. Igualmente, pode-se determinar o requerimento energético pela calorimetria indireta, sobretudo quando pairam dúvidas sobre a aplicabilidade ou precisão da equação de Harris-Benedict ${ }^{(5,11)}$.

Candidatos ao transplante com bom EN têm indicação de calorias em quantidade aproximadamente igual ao requerimento basal acrescido de $20 \%^{(8,9,17,33)}$. Em determinadas circunstâncias (desnutrição moderada a grave, infecções) este acréscimo pode ser de $50 \%$ a $75 \%$. Outros adotam simplesmente ministrar 30 a $35 \mathrm{kcal} / \mathrm{kg} / \mathrm{dia}$, elevando tais valores para $45 \mathrm{kcal}$ se a avaliação do EN, baseada na determinação 
do peso seco ou ideal demonstrar tratar-se de paciente desnutrido ${ }^{(9)}$. O Quadro 1 resume as necessidades de quilocalorias e de proteínas, glicídios e lipídios no pré-transplante e nas fases imediata e tardia do pós-transplante, com referência a variadas condições do estado nutricional e à presença de diabetes ou intolerância à glicose. No que diz respeito às proteínas, os valores diários variam segundo o estado evolutivo da hepatopatia. Em pacientes que apresentam história de encefalopatia, apesar do conhecimento de que a proteína endógena é mais importante para seu desencadeamento do que a exógena, é aconselhável baixar a oferta de proteínas para $70 \mathrm{~g} /$ dia. Segundo MALIAKKAL e BISTRIAN ${ }^{(17)}$, esse valor pode ser adotado na ausência de encefalopatia aguda, situação que demandaria o uso de uma fórmula enriquecida de aminoácidos de cadeia ramificada, na dosagem de 40 a $70 \mathrm{~g} /$ dia. Tal medida tem-se mostrado eficaz para compensar a incapacidade hepática no metabolismo dos aminoácidos resultantes do catabolismo muscular quando a hepatopatia é avançada, como sói acontecer com os candidatos a transplante. A tática de refeições pequenas e freqüentes e de uma pequena refeição antes de dormir visa minorar os efeitos de proteólise e gliconeogênese que os hepatopatas apresentam em períodos prolongados de jejum ${ }^{(17)}$.

Os cuidados na administração de sais minerais e vitaminas não são menos relevantes. Freqüentemente o ato cirúrgico é acompanhado da administração de grandes volumes de colóides e cristalóides, causadores de sobrecarga de fluidos que demanda cuidadosa correção. Hipocalcemia pode ser resultado da quelação do cálcio ionizado pela ação de altas doses de citrato veiculado nos produtos de transfusão sangüínea. Assim também, deve-se ter em conta que o novo órgão apresenta resistência inicial aos efeitos do hormônio paratireoidiano, razão pela qual pode ser crucial a necessidade de correção da hipomagnesemia.

Especialmente quando está presente a má absorção decorrente da hepatopatia crônica prolongada, cuidados especiais devem ser tomados com a suplementação de vitaminas lipossolúveis. Sua posologia, como a dos mais importantes micronutrientes, se apresenta no Quadro 2.

No pós-transplante os distúrbios eletrolíticos podem ser agravados por conta do efeito das drogas imunossupressoras (Quadro 3). A hipercaliemia pode ser decorrente da ação da ciclosporina ou do FK506, que são os imunossupressores preferidos, mas é necessário que se atente para outras alterações eletrolíticas como a alcalose metabólica resultante quer da infusão de bicarbonatos durante a fase anhepática do transplante, quer da transformação de citratos em bicarbonato pela ação do novo órgão, fato esse indicativo da capacidade funcional do enxerto.

QUADRO 1 - Necessidades nutricionais em macronutrientes no pré e pós-transplante hepático

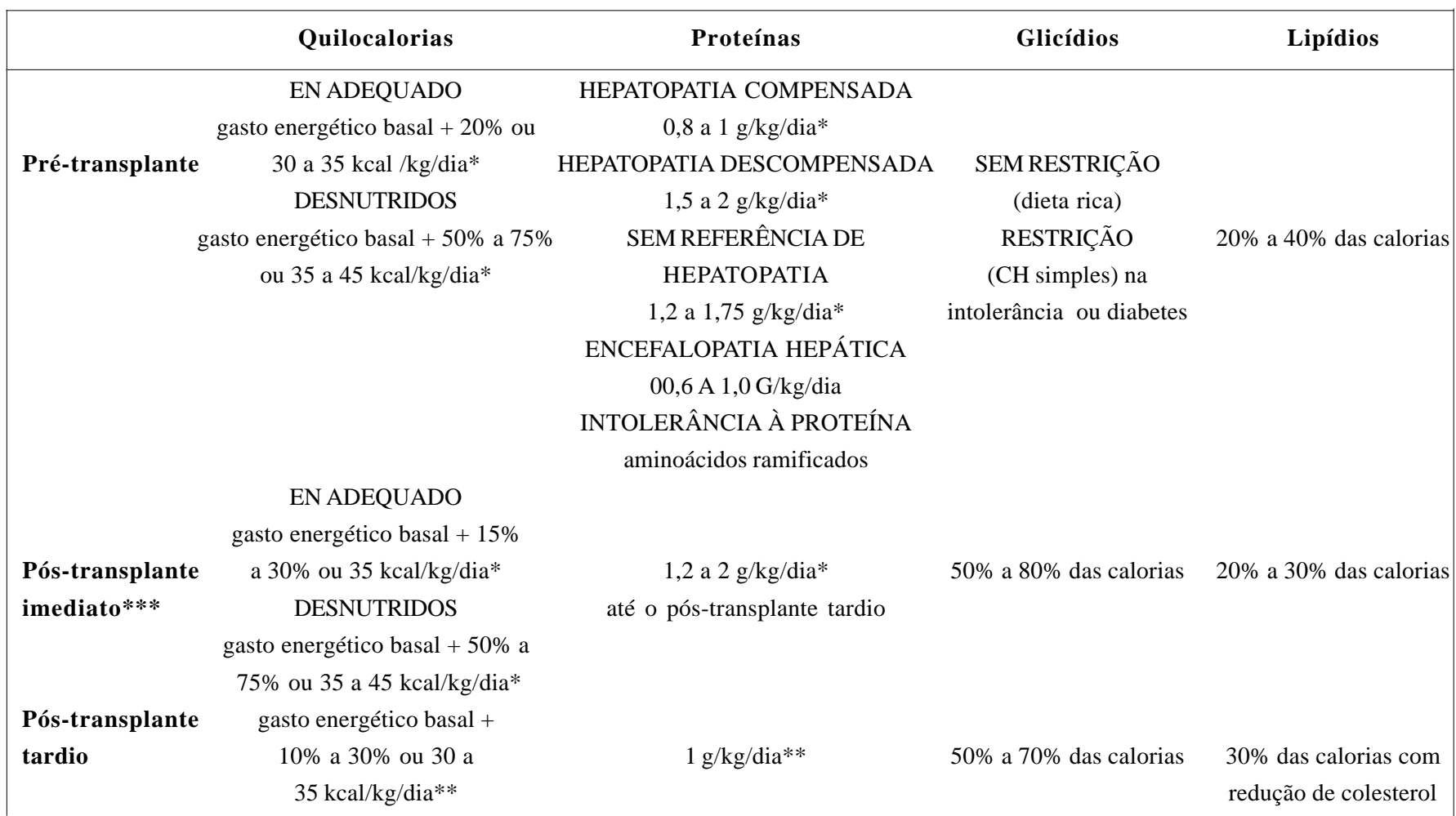

EN = estado nutricional $; \mathrm{CH}=$ carboidratos; $*$ peso seco ou ideal;** peso atual; $* * *$ até 2 meses de pós-operatório 
QUADRO 2 - Necessidades nutricionais em micronutrientes e líquido no pré e pós-transplante hepático

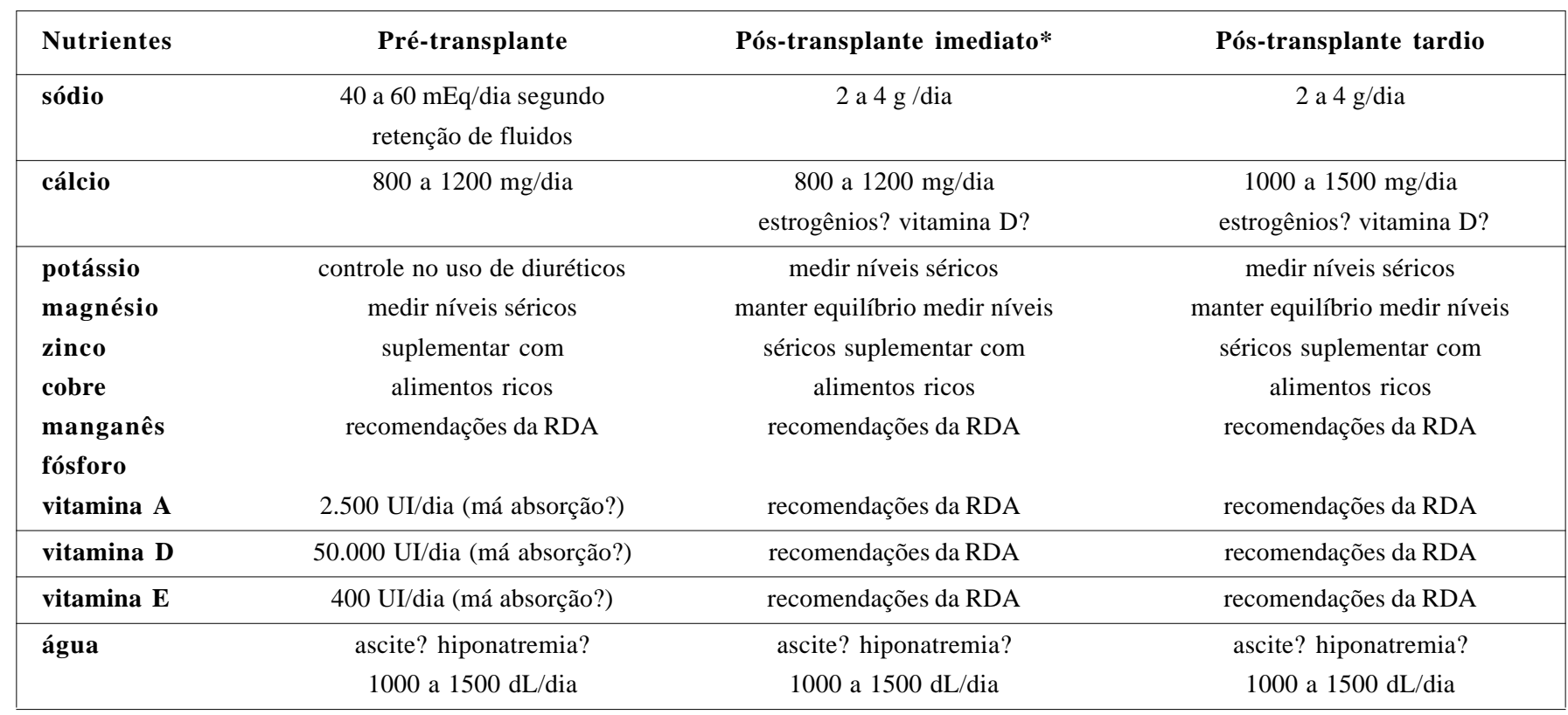

* Até 2 meses de pós-operatório

RDA = Recommended Dietary Allowance

QUADRO 3 - Efeitos nutricionais dos imunossupressores e sua correção

\begin{tabular}{|c|c|c|}
\hline Droga & Efeitos & Terapia \\
\hline Ciclosporina & $\begin{array}{c}\text { hiperlipidemia } \\
\text { hiperglicemia } \\
\text { hipercaliemia } \\
\text { hipomagnesemia }\end{array}$ & $\begin{array}{l}\downarrow \text { LIP e CH simples } \\
\qquad \text { CH simples } \\
\text { restrição de potássio } \\
\text { suplementar magnésio }\end{array}$ \\
\hline Glicocorticóides & $\begin{array}{c}\text { >catabolismo } \\
\text { <cicatrização } \\
\text { hiperlipidemia } \\
\text { hiperglicemia } \\
\text { retenção de sódio } \\
\text { hiperfagia } \\
\text { hipercalciúria }\end{array}$ & $\begin{array}{c}\uparrow \text { ingestão de proteína } \\
\downarrow \text { LIP e CH } \\
\downarrow \text { CH simples } \\
\downarrow \text { ingestão de sódio } \\
\text { regular quantidade } \\
\uparrow \text { ingestão } \mathrm{Ca}^{++} \text {ou suplementação }\end{array}$ \\
\hline Azatioprina & $\begin{array}{c}\text { anorexia, náusea, vômitos, dor } \\
\text { de garganta paladar alterado }\end{array}$ & $\begin{array}{l}\text { ajuste da dieta e do número } \\
\text { de refeiçõesmonitorar a ingestão } \\
\text { variedade de sabores e comidas }\end{array}$ \\
\hline OKT3 & $\begin{array}{l}\text { anorexia, náusea, } \\
\text { vômitos, diarréia }\end{array}$ & $\begin{array}{l}\text { ajuste da dieta e do número de } \\
\text { refeições monitorar a ingestão }\end{array}$ \\
\hline $\begin{array}{l}\text { Micofenolato de } \\
\text { Mofetil }\end{array}$ & diarréia & hidratação \\
\hline Tacrolimus & $\begin{array}{l}\text { diarréia } \\
\text { hiperglicemia } \\
\text { hipercaliemia } \\
\text { náusea, vômitos }\end{array}$ & $\begin{array}{c}\text { hidratação } \\
\downarrow \text { CH simples } \\
\downarrow \text { potássio } \\
\text { ajuste da dieta, monitorar a ingestão }\end{array}$ \\
\hline
\end{tabular}

LIP = lipídios; $\mathrm{CH}=$ carboidratos 


\section{METODOLOGIA}

Vários parâmetros são disponíveis para uma correta avaliação do estado nutricional, cabendo sua descrição sumária:

\section{Antropometria}

Os dados antropométricos devem ser relacionados ao sexo e à idade dos pacientes e compreendem: a determinação do índice de massa corporal (IMC) que é a relação entre o peso em quilogramas e o quadrado da estatura em metros $\left[\mathrm{kg} /(\mathrm{m})^{2}\right]$; a relação percentual entre o peso atual e o peso usual expressos em quilogramas $(\% \mathrm{~Pa} / \mathrm{Pu})$; a relação percentual entre o peso atual e o peso ideal, expressos em quilogramas $(\mathrm{Pa} / \mathrm{Pi})$; o percentual de gordura corporal, utilizando-se o somatório das medidas das pregas subescapular, supra-ilíaca, tricipital e bicipital; a adequação da prega cutânea tricipital em comparação à ideal, expressas em milímetros; a relação percentual das medidas tomadas em centímetros, da circunferência muscular do braço atual e ideal $(\mathrm{CMBa} / \mathrm{CMBi})$ que, embora mais acuradas que as avaliações de peso, são passíveis, também, de múltiplas causas de erros, não apenas devidos às observações e aos instrumentos, como às alterações que se verificam nos compartimentos corporais na doença hepática crônica.

Cabe ressaltar ainda, que na presença de ascite e edemas, as avaliações que dependem dos pesos (atual, ideal, seco), podem apresentar dificuldades de valor interpretativo, pois não é incomum encontrarem-se pacientes nessas condições, cujo peso atual seja menor que o peso ideal ou o peso seco.

A impedância bioelétrica, expressa em $\operatorname{Ohms}(\Omega)$ mede resistência e impedância elétrica entre dois pontos do corpo, com a finalidade de estimar a água corporal total ${ }^{(6)}$. Estudos comparativos com a aplicação de isótopos radioativos $\mathrm{H} 2$ [18O] mostram que a determinação da água total pela bioimpedância é inadequada em pacientes portadores de ascite ${ }^{(19)}$. A utilização da bioimpedância nessas circunstancias só é factível pelo emprego de programas adequados de correção.

\section{Estudo bioquímico}

A determinação de proteínas séricas (albumina, pré-albumina, transferrina, proteína transportadora de retinol) poderá apresentar resultados que dependem do estado de hidratação, da presença de insuficiência renal, de má absorção intestinal, do grau de lesão hepática, assim como do estoque de ferro e zinco e da ação de esteróides. $\mathrm{O}$ índice de creatinina relacionado à altura tem sido utilizado para a avaliação da massa muscular. Seu valor como parâmetro de avaliação depende da precisão na coleta da urina de 24 h, da presença de insuficiência renal e do grau de competência hepática para gerar creatina. Da mesma forma, o balanço nitrogenado está sujeito aos viéses representados pela falência renal e pelas dificuldades na coleta. A 3-metil-histidina, aminoácido liberado no catabolismo muscular, eliminado essencialmente pela urina, prestase à avaliação da perda de massa muscular nas hepatopatias crônicas. Entretanto, resultados falso-positivos decorrem de sua origem também extramuscular e da influência de vários fatores, como idade, sexo e o tipo de dieta adotada.

\section{Imunidade}

Como reflexo da higidez do EN, pode-se avaliar as condições de resposta imunitária, pela contagem absoluta de linfócitos e pelos testes cutâneos de sensibilidade tardia (PPD, Cândida). Entretanto, várias condições comumente presentes nas hepatopatias crônicas, como infecções, desequilíbrio hidroeletrolítico, insuficiência hepática ou renal, doenças inflamatórias intestinais, estresse metabólico e imunossupressão, podem influir na anormalidade dos resultados.

\section{Dinamômetro}

A utilização do dinamômetro fornece, de maneira indireta, informações sobre o EN, quando outros fatores capazes de determinar alteração da capacidade muscular não estão em jogo. $\mathrm{O}$ resultado desta avaliação pode revestir-se de valor prognóstico quanto à ocorrência de complicações pós-operatórias.

\section{Avaliação clínica}

$\mathrm{Na}$ avaliação global subjetiva, inclui-se a tomada da história nutricional do paciente, com registro de perdas de peso, alteração do apetite, saciedade precoce, alteração do paladar, sintomas gastrointestinais (diarréia, náusea, vômitos), hábitos dietéticos, adequação da ingestão de nutrientes e grau de atividade física. O exame físico permite a detecção de perda de massa muscular e adiposa, assim como a presença de ascite ou edemas.

A avaliação global já permite classificar o paciente em: bem nutrido, levemente desnutrido, moderadamente desnutrido e gravemente desnutrido, representando pois, o passo inicial para se definir grau e natureza da correção nutricional de pacientes candidatos ou submetidos ao transplante hepático ${ }^{(7,16,17,20,28,32)}$. O Quadro 4 resume algumas condições clínicas que podem estar presentes na desnutrição, correlacionáveis com alguma deficiência.

\section{TERAPIA NUTRICIONAL NO PÓS-TRANSPLANTE IMEDIATO}

No pós-transplante imediato o estado de catabolismo causado pela doença hepática que motivou o transplante é exacerbado pelo estresse da cirurgia, pelo uso de doses elevadas de esteróides e, com 
QUADRO 4 - Manifestações clínicas da desnutrição

\begin{tabular}{|lcc|}
\hline Órgão & Sinal ou sintoma & Possível deficiência \\
\hline $\begin{array}{l}\text { Cabelos } \\
\text { Olhos }\end{array}$ & secos e quebradiços & zinco \\
& cegueira noturna & vitamina A \\
& xerose, dermatite escamosa, & \\
vitaminas A e K, zinco, niacina & aumento de hematomas & \\
estomatite, queilose, glossite, & alteração de paladar & \\
Extremidades & irritabilidade neuromuscular, & riboflavina, vitamina B12, \\
& perda da sensibilidade e fraqueza & folato, magnésio, zinco \\
\end{tabular}

freqüência, pela presença de disfunção hepática, renal ou sepse ${ }^{(10,16)}$. Pacientes bem nutridos ou com desnutrição leve no pós-operatório e que estejam aptos a se alimentar pela via oral poucos dias após o transplante não necessitam de suporte nutricional específico. Iniciase dieta líquida a partir do $3^{\circ}$ ou $4^{\circ}$ dia de pós-operatório, progredindo para dieta livre, com alimentos cozidos, nos dias subseqüentes.

Nos pacientes incapazes de se alimentar pela via oral, ou quando se torna evidente a incapacidade de consumo de quantidades adequadas de nutrientes pela via oral, faz-se necessária a instalação de suporte nutricional especializado. Frente à dificuldade de se prever tal circunstância, o suporte nutricional pode ser instituído de maneira precoce, a fim de se evitar a desnutrição ${ }^{(8,9)}$.

Apesar da presença de ileogástrico e intestinal nos primeiros dias pós-transplante, a nutrição enteral pode ser administrada com segurança via sonda nasojejunal; além de menos dispendiosa que a parenteral total, a nutrição enteral oferece inúmeros benefícios como, menor risco de infecção, menor resposta metabólica ao estresse, menor risco de complicações técnicas e metabólicas, aumento da síntese protéica visceral e proteção contra a translocação bacteriana ${ }^{(9)}$. No período de transição da dieta enteral para oral, os alimentos podem ser administrados por sonda no período noturno, o que melhora o apetite e permite ao paciente maior movimentação durante o dia. A dieta enteral não deve ser interrompida antes que o paciente seja capaz de receber pela via oral dois terços a três quartos das necessidades nutricionais diárias. Quando complicações intra-abdominais e íleo paralítico prolongado impeçam a nutrição enteral, está indicada a instituição de nutrição parenteral total ${ }^{(10)}$.

\section{TERAPIA NUTRICIONAL NO PÓS-TRANSPLANTE TARDIO}

Enquanto o principal objetivo no pós-transplante imediato é a recomposição das reservas corporais, a terapia nutricional em longo prazo visa prevenir ou reduzir problemas comuns no pós-transplante tais como obesidade, hiperlipemia, hipertensão arterial, diabetes mellitus e osteoporose ${ }^{(10)}$.

\section{Obesidade}

O ganho excessivo de peso é problema freqüente e preocupante no pós-transplante tardio, atingindo até dois terços dos transplantados, variando de $20 \%$ a $40 \%$ a prevalência de IMC superior a $28 \mathrm{~kg} / \mathrm{m}^{2(10,22,30)}$. A maior parte do peso excessivo se adquire no primeiro ano após o transplante e é mais problemático nos pacientes sob esquema de imunossupressão com ciclosporina do que nos sob FK506. Dados apresentados em estudo multicêntrico demonstram obesidade em 18,2\% dos pacientes sob uso de ciclosporina contra $10,9 \%$ nos que utilizavam FK506 ${ }^{(27)}$. As causas são multifatoriais e incluem a recuperação do apetite com melhora do estado geral, a hiperfagia associada ao uso de corticóide e a manutenção da vida sedentária adquirida durante a doença ${ }^{(10)}$. Pacientes com história prévia de peso excessivo apresentam risco aumentado de desenvolver obesidade no pós-transplante(2)

A instalação precoce da terapia nutricional associada à atividade física representa a abordagem mais eficiente. Outras medidas incluem o uso de esteróides em dias alternados ou, eventualmente, sua retirada em pacientes com função estável do enxerto e sem evidências de rejeição.

\section{Hiperlipidemia}

A hiperlipidemia é achado comum em receptores de órgãos sólidos, incluindo os de transplante hepático. A frequiência geral de hipercolesterolemia em adultos submetidos ao transplante hepático varia de $17 \%$ a $43 \%$, enquanto a frequiência de hipertrigliceridemia é de aproximadamente $40 \%$, em relatos de diferentes centros ${ }^{(14)}$. A patogênese da hiperlipidemia é complexa e os principais fatores envolvidos incluem o efeito das drogas imunossupressoras, especialmente esteróides e ciclosporina, o desenvolvimento de diabetes mellitus após o transplante, a predisposição genética (do receptor e do doador), disfunção renal, ganho excessivo de peso e influências dietéticas ${ }^{(10,25,27)}$. 
Está bem estabelecido que as drogas promovam o desenvolvimento de hiperlipidemia no pós-transplante. Além dos conhecidos efeitos indesejáveis dos corticóides, a ciclosporina pode levar à hiperlipidemia pela inibição da síntese de ácidos biliares (inibição de 26-hidroxilase), pela ligação a receptores LDL, aumentando níveis séricos do colesterol LDL, pela indução de hiperinsulinemia, ou pela combinação de todos esses mecanismos ${ }^{(27)}$.

Vários estudos indicam que os esquemas de imunossupressão baseados na ciclosporina estão associados a níveis mais elevados de colesterol sérico do que aqueles que utilizam FK506 ${ }^{(2,13,14,26)}$. Estudo realizado por CANZANELLO et al. ${ }^{(2)}$ em receptores de transplante hepático constatou que a imunossupressão baseada na ciclosporina resultou em maior prevalência de hipercolesterolemia, hipertensão arterial e obesidade em comparação à obtida pelo uso de FK506.

\section{Hipertensão arterial}

A hipertensão arterial, infreqüiente na fase pré-transplante, ocorre em $20 \%$ a $60 \%$ dos pacientes em acompanhamento pós-transplante hepático, sendo discretamente mais prevalente nos obesos ${ }^{(25)}$.

O objetivo do tratamento da hipertensão arterial é reduzir a pressão arterial média, preservando simultaneamente a função renal e minimizando fatores de risco para doença cardíaca, como hiperlipidemia, distúrbios eletrolíticos e intolerância à glicose. A orientação nutricional tem papel relevante na correção desses fatores de risco, associada ao tratamento medicamentoso convencional com bloqueadores dos canais de cálcio que não interajam com a ciclosporina (nifedipina, isradipina, amlopidina). Apesar de não haver estudos que comprovem a efetividade das dietas restritivas de sódio no controle da hipertensão arterial nos receptores de transplante hepático, a recomendação é do consumo diário de $2 \mathrm{a} 4 \mathrm{~g}^{(10)}$.

\section{Diabetes mellitus}

Hiperglicemia é problema comum em pacientes transplantados e está relacionada aos efeitos diabetogênicos intrínsecos da ciclosporina e do FK506, que incluem redução da secreção de insulina, aumento da resistência a este hormônio e, possivelmente, efeito tóxico direto sobre as células $\beta$ das ilhotas pancreáticas ${ }^{(35)}$. Tais efeitos são agravados pela ação dos corticóides utilizados nos esquemas de imunossupressão.

A hiperglicemia que ocorre no período pós-operatório imediato, pode comportar-se como simples intolerância transitória à glicose que necessita o uso temporário de insulina. A minoria dos pacientes requer seu uso permanente. MUÑOZ et al. ${ }^{(25)}$ relatam maior incidência de diabetes em pacientes obesos (33\%) comparados aos não-obesos $(17 \%)$. Controle dietético e de peso, exercícios regulares e insulina, quando necessária, são a base do tratamento.

\section{Osteoporose}

A osteoporose é complicação freqüente e muitas vezes severa nos transplantados de órgãos, podendo afetar significativamente a qualidade de vida dos pacientes. Cerca de $90 \%$ dos submetidos a transplante hepático apresentam perda da massa óssea nos 3 a 6 primeiros meses após a cirurgia. Vários fatores de risco contribuem para a osteoporose: uso de corticóides (perda acelerada de osso trabecular), doença óssea prévia ao transplante (na doença hepática crônica, especialmente cirrose biliar primária e colangite esclerosante), sedentarismo prolongado, hipogonadismo, perda de massa muscular e nutrição inadequada ${ }^{(33)}$.

Passado o período inicial de catabolismo ósseo, a tendência é de recuperação da massa óssea, diretamente proporcional à osteopenia pré-existente e à boa função do enxerto hepático. Fatores reversíveis como deficiência de cálcio e vitamina D devem ser prontamente identificados para a suplementação dietética, sendo importante, também, o estímulo a atividades físicas, adaptadas a condições individuais dos pacientes, a fim de se evitar complicações músculoesqueléticas.

Mulheres que apresentem osteopenia ou osteoporose após a menopausa se beneficiam com o uso de estrogênios. Parece promissor o uso de drogas inibidoras da reabsorção óssea, como os bifosfonados ${ }^{(12)}$.

Parolin MB, Zaina FE, Lopes RW. Nutritional therapy in liver transplantation. Arq Gastroenterol 2002;39(2):114-122.

ABSTRACT - Background - Malnutrition, sometimes severe is common in patients with chronic hepatic diseases who are candidates for liver transplantation. Nutritional therapy can induce partial or total correction of such deficiencies, improving clinical conditions and prognosis of patients who face the great defiance of liver transplantation. Aims - Brief revision of hepatic role in the metabolism of several nutrients. Description of available methods of dietary therapy and its application both under different abnormal hepatic conditions and pre and posttransplant periods. The role of nutritional intervention in metabolic side effects due to immunosuppressive drugs. Conclusion - Nutritional therapy is a valuable adjuvant resource to the clinical treatment of candidates and submitted patients to hepatic transplantation providing better prognosis and improved life quality.

HEADINGS - Liver transplantation. Liver failure, diet therapy. Liver diseases, diet therapy. Diet therapy. Nutritional status. 


\section{REFERÊNCIAS BIBLIOGRÁFICAS}

1. Akerman PA, Jenkins RL, Bistrian BR. Preoperative nutrition assessment in liver transplantation. Nutrition 1993; 9:350-6.

2. Canzanello V, Schwartz L, Taler S, Textor S, Wiesner R, Porayko M, Krom R. Evolution of cardiovascular risk after liver transplantation. A comparison of cyclosporine A and tacrolimus(FK506). Liver Transplant Surg 1997;3: 1-9.

3. Crawford, DHG, Cuneo RC, Shepherd RW. Pathogenesis and assessment of malnutrition in liver disease. J Gastroenterol Hepatol 1993;8:89-94.

4. Dicecco SR, Wieners EJ, Wiesner RH, Southorn PA, Plevak DJ, Krom RAF. Assessment of nutritional status of patients with end-stage liver disease undergoing liver transplantation. Mayo Clin Proc 1989;64:95-102.

5. Doltz C, Raurich JM, Ibanez J, Obrador UM, Marse P, Gaya J. Ascitis increases the resting energy expenditure in liver cirrhosis. Gastroenterology 1991;100:738-44

6. Guglielmi FW, Contento F, Laddagal L, Panella C, Francavilla A. Bioelectric impedance analysis: experience with male patients with cirrhosis. Hepatology 1991;13:892-95

7. Hasse JM. Nutritional implications of liver transplantation. Henry Ford Hosp Med J 1990;38:235-40.

8. Hasse JM, Blue LS, Crisppin JS, Goldstein RM, Jennings LW, Gonwa JA, Husberg BS, Levy MF, Klintmalm GB. The effect of nutritional status on length of stay and clinical outcomes following liver transplantation. J Am Diet Assoc 1994; suppl A-38.

9. Hasse JM, Blue LS, Liepa GU, Goldstein RM, Jennings LW, Mor E, Husberg BS, Levy MF, Gonwa TA, Klintmalm GB. Early enteral nutrition support in patients undergoing liver transplantation. JPEN J Parenter Enteral Nutr 1995;19:437-43.

10. Hasse J. Nutritional aspects of adult liver transplantation. In: Busutil RW, Klintmalm GB, editors. Transplantation of the liver. Philadelphia: WB Saunders; 1996. p.359-67.

11. Hasse JM, Weseman B, Fuhrman MP, Loeffler M, Francisco Ziller N, Dicceco SR Nutrition therapy for end stage liver disease: a practical approach. Support Line 1997; $19: 8-15$.

12. Hay JE. Osteoporosis. Clin Liver Dis 1998;2:407-19.

13. Imagawa D, Dawson S, Holt C, Kirk P, Kaldas F, Shackleton C, Seu P, Rudich SM, Kinkhabwala MM, Martin P, Goldstein LI, Murray NG, Terasaki PI, Busutil RW. Hyperlipidemia after liver transplantation: natural history and treatment with the hydroxymethylglutaryl-coenzyme A reductase inhibitor pravastin. Transplantation 1996;62:934-42.

14. Jindal R, Popescu E, Emre S, Schwartz M, Boccagni P, Meneses P, Mor E, Sheiner $\mathrm{P}$, Miller CM. Serum lipid changes in liver transplant recipients in a prospective trial of cyclosporine versus FK506. Transplantation 1994;57:1395-8.

15. Kalman RD, Saltzman JR. Nutrition status predicts survival in cirrhosis. Nutr Rev 1996;54:217-9.

16. Lowell JA. Nutritional assessment and therapy in patients requiring liver transplantation. Liver Transplant Surg 1996;2(Suppl 1):78-88.

17. Maliakkal RJ, Bistrian BR. Improving nutritional status in patients with chronic liver disease. J Crit Illness 1993;8:169-82.
18. McCullough AJ, Mullen KD, Smanik EJ, Tabbaa M, Szauter K. Nutritional therapy and liver disease. Gastroenterol Clin North Am 1989;18:619-43.

19. McCullough AJ, Mullen KD, Kalhan SC. Measurements of total body and extracellular water in cirrhotic patients with and without ascites. Hepatology 1991;14:1102-11

20. McCullough AJ, Teran JC, Burgianesi E. Guidelines for nutritional therapy in liver disease. ASPEN, 1998. p.1-12.

21. McCullough AJ. Malnutrition and liver disease. Liver Transplant 2000;6(4 Suppl):S85-S96

22. Mor E, Facklam D, Hasse J, Sheiner P, Emre S, Schwartz M, Miller C. Weight gain and lipid profile in liver transplant recipients: long-term results of the American FK506 Multicenter Study. Transplant Proc 1995;27:1126.

23. Müller MJ, Lautz HU, Plogmann B, Bürger M, Körber J, Schmidt FW. Energy expenditure and substrate oxidation in patients with cirrhosis: the impact of cause, clinical staging and nutritional state. Hepatology 1992;15:782-94.

24. Müller MJ, Bötcher J, Selberg O, Weselmann S, Böker KHW, Schwarze M, von zur Mühlen A, Manns MP. Hypermetabolism in clinically stable patients with liver cirrhosis. Am J Clin Nutr 1999;69:1194-201.

25. Muñoz SJ, Deems RO, Moritz MJ, Martin P, Jarrell BE, Maddrey WC. Hyperlipidemia and obesity after orthotopic liver transplantation. Transplan Proc 1991;21:1480-3.

26. Muñoz S. Hyperlipidemia and coronary risk factor after orthotopic liver transplantation: pathogenesis, diagnosis, and management. Liver Transplant Surg 1995;1(Suppl1):29-38.

27. Muñoz SJ. Progress in postransplant hyperlipidemia. Liver Transplant Surg 1997;3:439-42.

28. Nompleggi DJ, Bonkowsky HL. Nutritional supplementation in chronic liver disease: an analytical review. Hepatology 1994;19:518-33.

29. Olson RE. Vitamin K. In: Goodhart RS, Shils ME, editors. Modern nutrition in health and disease. 6.ed. Philadelphia: Lea \& Febiger; 1980. p.170-80.

30. Palmer M, Schaffner F, Thung SN. Excessive weight gain after liver transplantation. Transplantation 1991;51:797-800.

31. Pikul J, Sharpe MD, Lowndes R, Chen CN. Degree of preoperative malnutrition is predictive of postoperative morbidy and mortality in liver transplant recipients. Transplantation 1994;57:469-72.

32. Porayko MK, Dicecco S, O'Keefe SJD. Impact of malnutrition and its therapy on liver transplantation. Sem Liver Dis 1991;11:305-14.

33. Porayko MK, Wiesner RH, Hay HE, Krom RAF, Dickson ER, Beaver S, Schwerman L. Bone disease in liver transplant recipients: incidence, timing, and risk factors. Transplant Proc 1991;23:1462-5.

34. Seifer S, Englard S. Energy metabolism. In: Arias IM, Boyer JL, Fausto N, Jakoby WB, Schachter DA, Shafritz DA, editors. The liver biology and pathobiology. 3.ed. New York: Raven Press; 1944. p.323-64.

35. Testa G, Klintmaln GB. Cyclosporine and tacrolimus. The mainstay of immunosuppressive therapy for solid organ transplantation. Clin Liver Dis 1997;1:417-37.

Recebido em 10/4/2001 Aprovado em 16/8/2001. 